Voix et Images

volxetimages

\title{
Poésie impure, poésie/prose, classicisme
}

\section{André Brochu}

Volume 41, numéro 2 (122), hiver 2016

URI : https://id.erudit.org/iderudit/1036944ar

DOI : https://doi.org/10.7202/1036944ar

Aller au sommaire du numéro

Éditeur(s)

Université du Québec à Montréal

\section{ISSN}

0318-9201 (imprimé)

1705-933X (numérique)

Découvrir la revue

Citer ce compte rendu

Brochu, A. (2016). Compte rendu de [Poésie impure, poésie/prose, classicisme].

Voix et Images, 41(2), 139-145. https://doi.org/10.7202/1036944ar d'utilisation que vous pouvez consulter en ligne.

https://apropos.erudit.org/fr/usagers/politique-dutilisation/ 


POÉSIE
Poésie impure, poésie/prose, classicisme
+++

ANDRÉ BROCHU

Université de Montréal

Au programme, deux essais sur la poésie et, au milieu, un recueil qualitativement et quantitativement important.

L'abbé Henri Bremond, contemporain de Paul Valéry, était un grand amateur de littérature, doublé d'un remarquable critique et d'un théoricien du verbe. Il recherchait dans les œuvres une dimension idéale que son inclination religieuse jugeait supérieure à tout. Il a donc créé le concept de poésie pure, qu'il appliquait aux réussites d'ordre lyrique. Pour lui, affirme un commentateur récent, il y a quelque «impureté métaphysique» à «enseigner, raconter, peindre, donner le frisson ou tirer des larmes ${ }^{1}$ ». C'est là l'effet ou la mission de la prose (animus), et la poésie (anima) doit s'en garder, elle qui est vouée à l'inanalysable, à quelque mystère nodal du texte.

$C^{\prime}$ est contre une telle conception, passablement répandue encore de nos jours, que s'élève Robert Melançon dans son dernier ouvrage ${ }^{2}$. Comme s'il reprenait, en la développant mais pour la contredire, l'énumération citée plus haut, il affirme: «Un poème peut se faire méditation, inventaire, lettre, gémissement, cri de joie, babillage, raisonnement, éloge, vitupération, journal intime, éditorial.» (11) La poésie n'est pas la prose, mais elle ne peut se passer d'elle, car la prose est seule capable de lui faire tremplin pour l'élever jusqu'à la hauteur désirée.

Cependant, on aurait tort de penser que Melançon, excellent poète lui-même et critique averti, favorise le laisser-aller de l'inspiration et de l'expression. Il suffit de lire les études qu'il consacre à un Robert Marteau (119-125) ou à un Jacques Réda (127-140) pour constater le culte de la rigueur qui étaie l'admiration qu'il leur voue. La précision métrique notamment, pourtant exercée en contexte de modernité donc de vers libres, fait justement de ces derniers « véritablement des vers, non de la prose en morceaux» (131). Rappelons que Melançon lui-même, dans son admirable Paradis des apparences ${ }^{3}$, équilibre en quelque sorte le réalisme de l'inspiration par la rigueur

1 Jacques Jouet, «Poésie pure», Encyclopædia Universalis, en ligne: http://www.universalis.fr/encyclopedie/ poesie-pure (page consultée le 13 avril 2016).

2 Robert Melançon, Pour une poésie impure, Montréal, Boréal, coll. «Papiers collés», 2015, 206 p.

3 Robert Melançon, Le paradis des apparences. Essai de poèmes réalistes, Montréal, Éditions du Noroît, 2004, $150 \mathrm{p}$. 
métrique, alignant cent quarante-quatre $(12 \times 12)$ poèmes de douze vers (proches de l'alexandrin - vers de douze pieds).

En quoi consiste donc l'impureté salvatrice que l'auteur appelle de ses vœux? On voit bien qu'elle tient non pas à la forme, qui se veut idéale, mais à un contenu contraire à celui de la poésie romantique, celle qui se veut inspirée et étrangère au quotidien. Robert Melançon me semble ranimer en notre époque ce mouvement qui a récusé le romantisme au profit d'une poésie plus terre à terre, le Parnasse, qu'on peut définir précisément en termes d'impureté (à distinguer, bien entendu, de l'abjection ou de la saleté). Le Parnasse dit l'immédiat, le directement saisissable, non sollicité par quelque rêve infini. Certes, l'évocation du réel peut et doit être singulière. Prenons ce passage de Robert Marteau, cité par Melançon:

\footnotetext{
Quelle grâce du moindre oiseau à la poursuite

D'un insecte en l'air qui va par à-coups et voltes

À dix degrés de l'horizon oriental. (119)
}

Certes, l'oiseau poursuivant l'insecte constitue un motif simple et relativement peu inspirant ou inspiré, la quête de nourriture rendant le vol prosaïque; mais la métrique (ici, trois alexandrins) et la formulation recherchée (voltes, dix degrés, horizon oriental) transforment l'image sur la base même de son «impureté» ou, si l'on veut, de sa banalité. Même chose dans ces vers de Jacques Réda, poète que Melançon met audessus de beaucoup d'autres:

On n'entend que les pattes des pigeons au bord des toits

Et le clapotage de l'eau contre la pierre du bassin;

À paisible cadence un balai chuchote sous la voûte. (129)

Voilà une menue scène urbaine centrée sur des êtres animés ou des choses en mouvement tout ordinaires (pigeons, clapotis, balayeur), aux antipodes de la vie affective telle que la peut éprouver le moi. La poésie ici surgit à l'intersection des choses et des mots qui les énoncent avec une parfaite et rare élocution. Le rapprochement se fait sans peine avec les «impures» et savoureuses évocations du Paradis des apparences:

Une bande lilas, que coupe l'alignement des maisons,

Que cloisonnent les ramures d'hiver,

Tient lieu de crépuscule dans les carreaux. (60)

La poésie de ces vers tient simultanément de la réalité du représenté (le recueil est sous-titré «Essai de poèmes réalistes») et de la façon unique de le dire.

Les études rassemblées dans Pour une poésie impure n'abordent pas toutes, directement du moins, la question de l'impur. Elles tendent à le faire, mais n'y parviennent pas toujours. Par exemple, un ensemble de textes est consacré à un poète bien-aimé, Saint-Denys Garneau, qui n'a rien, que je sache, d'un parnassien. L'auteur affirme que les lecteurs et commentateurs voient le plus souvent en lui « un symptôme de l'aliénation canadienne-française», des «misères psychologiques engendrées par 
une prétendue impuissance à vivre» (25). Voilà qui ressemble fort à la problématique romantique. Melançon découvre plutôt dans cette œuvre une «expérience religieuse authentique» (25), une «aventure intérieure menée à ses ultimes conséquences avec une intransigeance héroïque» (25-26). En somme, une vérité profonde qui a le caractère et la solidité d'une substance fonde l'élan poétique garnélien, en accord avec une quotidienneté compacte sinon impure, au sens strict du mot...

Là où le texte poétique se donne le plus immédiatement et totalement comme impur, c'est dans le haïku, court poème de trois lignes seulement dont André Duhaime est, dans nos lettres, le principal représentant. «[I]l n'existe sans doute pas d'art plus réaliste» (192), affirme Melançon à propos de cette exaltation du concret qu'est le bref poème d'origine japonaise, qui dit «les choses, les gens, les bêtes, les plantes, les fêtes du calendrier, les saisons, les éléments, le temps qu'il fait, les paysages, des gestes, de menus accidents[;] tout ce qui tisse l'étoffe chamarrée du monde lui offre une matière inépuisable» (193).

On voit l'originalité de la perspective adoptée par l'auteur, qui ne se fait pas faute de prendre le contre-pied des positions de la critique ou de la poétique actuelles. À l'opposé de la conception courante de la poésie, qui fait d'elle une affaire de mystère souvent facile et de sentimentalité, Melançon défend l'idée d'une appropriation du réel, d'une assumation du concret et, par là, du monde même. C'est ainsi qu'il oppose aux premiers recueils de son ami Jacques Brault, marqués par une «ampleur incantatoire» (156), ceux qui ont suivi et qui présentent «quelque chose de familier, proche, immédiat» (157), qui disent «le quotidien dans ce qu'il a de moins extraordinaire» (159).

À la limite, sans doute, la démarche risque de nous conduire au silence, par un trop grand respect du non-mystère. Ce n'est pas un hasard si, dans sa conclusion, Melançon manifeste son inquiétude devant le sort de la poésie qui se publie maintenant, poésie sans doute fort abondante mais de plus en plus délaissée des lecteurs, ce qui fait d'elle «un art en crise, précaire et menacé» (200). Notons que les poètes favoris de Melançon sont souvent peu lus, tel Jacques Réda («Il y a quarante ans, Réda devait compter environ trois cents lecteurs. Aujourd'hui? On sait son nom. Le lit-on beaucoup plus?» [140]).

On peut se demander si l'«impureté» aux antipodes de l'engagement littéraire, telle que la préconise l'auteur, peut ramener le lecteur au poème.

Dans le sujet suivant, qui traite d'un recueil ${ }^{4}$ et non d'un essai, s'agit-il de poésie pure ou impure? L'écriture de René Lapierre tient sans doute de l'une et de l'autre. Mais on pourrait parler plutôt d'un contrepoint de poésie et de prose, même si le récit, assez abondant, dissimule une intention évocatoire.

Le réel y est présent, bien qu'il se dévoile rarement sous des allures triviales ou anecdotiques; et un lyrisme sobre, qui s'emploie à dire les plus substantielles vérités du monde, lui tient compagnie.

4 René Lapierre, La carte des feux, Montréal, Les Herbes rouges, 2015, 224 p. 
Dieu (sans majuscule) apporte au sens lyrique sa touche d'infini. J'avais relevé déjà chez l'auteur ce particularisme étonnamment peu moderne (présent aussi chez d'autres contemporains pourtant dits d'avant-garde) dans un précédent recueil ${ }^{5}$.

On observe d'ailleurs une continuité remarquable entre les divers écrits de René Lapierre. Par exemple, deux "personnages» de L'eau de Kiev ${ }^{6}$ Paschetti et Solomon, qui relèvent aussi bien de la narration que du lyrisme, refont surface dans La carte des feux. On remarque également une disposition textuelle fort semblable dans un recueil comme Aimée soit la honte ${ }^{7}$ et le dernier paru: alternance de courts poèmes en italique et de poèmes plus longs, aux allures de prose. Mais c'est surtout l'esprit des textes qui peut frapper le lecteur. D'un recueil à l'autre, une même interrogation, aux accents simples et profonds, sollicite le lecteur et le met en présence des aspects cardinaux du monde: l'amour, la matière, l'esprit, le temps, la mort. On a l'impression que l'auteur, à partir d'éléments passablement précis sinon courants de l'existence (comme ceux relatifs au bizarre Pascetti), s'ingénie à s'élever bien au-delà de la vie quotidienne pour poser la question de l'être même, sous ses facettes les plus fondamentales:

\author{
Humain est celui \\ qui blesse et l'a voulu; \\ qui aime \\ et ne l'a pas voulu. \\ Humaine est notre catastrophe \\ humaine notre humanité. \\ À rien d'autre \\ nous ne la mesurons. (178)
}

L'homme apparaît ici comme le titulaire des solutions malfaisantes - la méchanceté est son lot. Pourtant il aime, mais cela se fait sans qu'il l'ait recherché. Le positif et le négatif en lui se côtoient selon un plan qui ne relève en rien de la volonté. Telle est la métaphysique qui régit l'humain: une catastrophe sans mesure. Et de nombreux passages affirment ce côté calamiteux de tous et de tout, à commencer par celui du moi lui-même: «Je suis l'anthracite, le poison/de ricin, le capital qui tue/ qui viole et qui jouit, avide/de profit et de mort.» (22) Le moi semble assumer tous les maux du monde, lesquels sont par ailleurs énumérés selon les divers niveaux où ils se présentent:

Niveau quatre: nous tolérons les génocides.

Les meurtres d'État, les guerres.

Nous inventons des formes générales

d'amnésie. (115)

5 René Lapierre, Love and Sorrow, Montréal, Les Herbes rouges, 1998, 72 p. Voir ma chronique intitulée «Poignées d'herbes rouges», Voix et Images, vol. XXIV, n 1, automne 1998, p. 203-206. Y sont cités également Jean-Marc Desgent et André Roy.

6 René Lapierre, L'eau de Kiev, Montréal, Les Herbes rouges, 2006, 151 p.

7 René Lapierre, Aimée soit la honte, Montréal, Les Herbes rouges, 2010, 102 p. 
La vaste énumération des maux individuels et collectifs, dont la liste rappelle les multiples visages d'un dieu rabaissé au stade anthropique («Mon dieu est un communiqué de presse, un égratigneur de vélos, un livre trop cher, une carte à jouer [...].» [84]), laisse pourtant de côté la catastrophe que la quatrième de couverture présente comme le sujet même du livre, cette dérive de notre continent il y a trois cent millions d'années, sous le nom de Laurentie. En fait, on pourrait sans doute retrouver à divers endroits du recueil des allusions à cet événement géographique majeur, mais elles passent inaperçues tant on s'attend à un récit caractérisé et détaillé. Les déambulations de Paschetti ou de Solomon sont de peu de poids à côté de l'importance qu'auraient revêtue les mouvements de la Terre.

Sans doute ne s'agit-il pas d'une faute de narration mais de la création d'une attente, chez le lecteur, susceptible de réfléchir l'immense drame d'une humanité lacunaire.

Un ouvrage curieux et, du reste, intéressant, vient de paraître sous la plume de Claude Paradis ${ }^{8}$. Il porte sur ce que celui-ci considère comme les classiques de notre poésie. Il en étudie dix, d'auteurs tous différents. Dans l'ordre où ils sont abordés, il s'agit de: Hector de Saint-Denys Garneau, Jacques Brault, Gaston Miron, Roland Giguère, Anne Hébert, Alain Grandbois, Jean-Aubert Loranger, Paul-Marie Lapointe, Robert Melançon et Pierre Morency. Par «classiques», Claude Paradis entend des recueils non seulement de haut niveau, mais magnifiquement composés, présentant presque la rigueur de romans ou de récits (il appelle d'ailleurs «chapitres» les sections d'un recueil). Un tel critère de composition, qui suppose une sorte de progression thématique interne, voire de rigueur narrative, confère à chacun des poèmes une résonance à l'échelle de l'ouvrage entier.

Pour cette raison, un auteur comme Nelligan, malgré son importance reconnue de tous, est exclu de la recherche, lui qui n'a pas réussi à boucler un livre bien à lui ! Les morceaux des anthologies sont également mis de côté, même si elles réunissent en principe les poésies les plus réussies. Pas de réussite, affirme Claude Paradis, hors la succession d'un ensemble complet et bien structuré, qui soit le fait d'un même auteur. C'est ainsi que Regards et jeux dans l'espace ${ }^{9}$, de Saint-Denys Garneau, est salué comme le plus grand chef-d'œuvre de notre poésie - et pas un mot sur les poèmes des Solitudes, réalisation posthume du même auteur, dont nous devons la publication à ses amis. Pourtant le poète français Alain Bosquet, auteur d'une précieuse anthologie de notre poésie ${ }^{10}$, a choisi presque tous les textes de Garneau dans Les solitudes. De Regards et jeux dans l'espace, il ne retient que "Faction", d'ailleurs très proche des poèmes publiés à titre posthume.

8 Claude Paradis, Ouvrir une porte sur dix grandes œeuvres de la poésie québécoise du Xxe siècle, Montréal, Éditions du Noroît, coll. «Chemins de traverse», 2015, 240 p.

9 Hector de Saint-Denys Garneau, Poésies complètes. Regards et jeux dans l'espace. Les Solitudes, Montréal, Fides, coll. «du Nénuphar», 1949, 227 p.

10 Alain Bosquet, La poésie canadienne, Paris/Montréal, Éditions Seghers/Éditions HMH, coll. «Melior», 1962, 222 p. Les poèmes de Saint-Denys Garneau sont reproduits des pages 47 à 60. 
Claude Paradis non seulement privilégie les recueils bien charpentés, mais il met beaucoup l'accent sur la dimension autobiographique, comme si celle-ci était garante de la réussite littéraire. L'oiseau, motif fréquent chez Garneau, est interprété comme un symbole majeur du poète lui-même - qui est «l'enfant-poète-oiseau» (42) -, et il en va de même chez Jacques Brault - qui «associe le cri de l'oiseau [bien sûr, un oiseau!] à la solitude que lui-même éprouve au même moment» (64) —, de sorte que toutes les significations, toutes les images se rapportent, en fin de compte, aux auteurs eux-mêmes. Les amours évoquées dans Moments fragiles de Jacques Brault ${ }^{11}$ seraient la matière immédiate de confidences personnelles, et non des expressions objectivement valables de la passion. Ou plutôt, les unes constitueraient les autres. Dans le chapitre sur Alain Grandbois ${ }^{12}$, la dimension autobiographique, voire amoureuse, est également très marquée, contrairement à ce que présentent les lectures habituelles.

Cela dit, les partis pris du critique n'empêchent pas, à l'occasion, des lectures judicieuses et novatrices, qui ont surtout l'avantage de donner à réfléchir sur des pans de l'œuvre restés jusque-là dans l'ombre. Par exemple, la figure de la femme aimée au cœur de "La marche à l'amour», dans le célèbre recueil L'homme rapaillé13 de Gaston Miron, devient l'incarnation même du pays: «le poète amalgame [...] l'engagement d'amour à l'engagement politique» (91), ce qu'on n'avait jamais souligné d'aussi claire façon. Par ses rapprochements, Paradis fait apparaître d'éclairantes ressemblances non seulement entre des thèmes d'un même recueil, mais aussi entre des recueils différents. Par exemple, Anne Hébert et Jacques Brault apparaissent parfois comme les sosies de Saint-Denys Garneau, ce qui renforce la dimension «classique» de l'une et de l'autre, comme s'ils participaient d'une même inspiration.

Plus loin, Claude Paradis loue sans réserve les deux versions, pourtant fort différentes, de Peinture aveugle de Robert Melançon ${ }^{14}$, dans lesquelles il retrouve une poésie simple et totale, aux antipodes du formalisme des années 1970 - cet antiformalisme, c'est peut-être bien cela, l'impureté si haut proclamée!

Quelques-uns des livres qu'analyse Claude Paradis sont moins proches de la poétique garnélienne. Alain Grandbois, Roland Giguère et Paul-Marie Lapointe s'éloignent de l'individualisme et de l'intimisme communs aux autres - notamment, en plus de ceux dont j'ai déjà parlé, un Jean-Aubert Loranger et une Anne Hébert.

Claude Paradis conclut son livre par l'analyse de Pierre Morency, chez qui l'on retrouve des éléments de représentation proches de ceux rencontrés chez plusieurs des auteurs précédents, et qui étonnent par leur absence de lyrisme:

Êtes-vous de ceux qui tuent les araignées dans les maisons?

[...] Êtes-vous de ces personnes qui pensent à l'argent plus de dix minutes par jour?

(211)

11 Jacques Brault, Moments fragiles, dans Poèmes, Montréal, Éditions le Noroît, coll. «Ovale», 2000, p. 267350.

12 Alain Grandbois, Les îles de la nuit, dans Poèmes, Montréal, l’Hexagone, 1963, p. 9-96.

13 Gaston Miron, L’homme rapaillé, Montréal, TYPO, 1993, 262 p.

14 Robert Melançon, Peinture aveugle, Montréal, VLB éditeur, 1979, 88 p. et Peinture aveugle, édition revue, Montréal, Éditions du Noroît, 2010, 68 p. Robert Melançon a remanié profondément son recueil même si celui-ci avait mérité en 1979 le Prix du Gouverneur général du Canada. 
On cherche en vain la poésie de telles propositions, tout comme celle de bien des poésies «impures» qui se cantonnent platement, pourrait-on dire, dans la description d'éléments du paysage. On est alors bien loin des denses évocations du sacré ou de l'humain chez une Rina Lasnier ou un Fernand Ouellette, ou des revendications ferventes d'un Paul Chamberland. 